\title{
Influence des déformations sur la mesure des caractéristiques élastiques de roches tendres
}

\section{F. SKOCZYLAS}

École Centrale de Lille-

URA CNRS 1441,

Cité Scientifique BP 48,

59651 Villeuneuve-d'Ascq

Cedex

\section{J.-P. HENRY}

Laboratoire de Mécanique de Lille-URA CNRS 1441, 59655 Villeneuve-d'Ascq

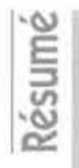

Pour déterminer les caractéristiques élastiques de matériaux rocheux tendres ou très déformables, telles que les craies, il est habituel de procéder à des cycles de déchargements élastiques. Ces déchargements sont parfois effectués quand la déformation du matériau a atteint un niveau que l'on ne peut qualifier

d'infinitésimal. Ce procédé est également employé pour étudier la variation des modules élastiques d'un matériau endommageable qui peut aussi être très déformable (certains sels par exemple). La modélisation de tels matériaux nécessite alors la connaissance précise de ces modules élastiques que la simple lecture d'une pente de décharge ne peut fournir du fait des changements de géométrie de l'échantillon testé. Nous proposons donc d'étudier dans cet article, la démarche qui permet de corriger les mesures conventionnelles des paramètres élastiques d'un échantillon cylindrique soumis à une sollicitation triaxiale en cellule de confinement. La géométrie axisymétrique du problème permet d'obtenir aisément le gradient de la transformation et de revenir par son intermédiaire à l'expression du tenseur des contraintes de Piola-Kirchhoff et des déformations lagrangiennes qui lui seront reliées. Cette étude reprend enfin quelques résultats obtenus sur une craie blanche, au Laboratoire de Mécanique de Lille, dans le but de montrer l'ampleur des corrections qu'il y aurait lieu d'effectuer dans la détermination du coefficient de Poisson et du module élastique d'un matériau isotrope.

\section{Elastic characteristics measurement of low strength rocks in the case of large strain}

In some circumstances, elastic characteristics of low strength material, such as chalk, are measured by elastic unloading cycles. Sometimes unloading occurs when strains are larcie, of the order unity. Such situations fall outside the scope of classical interpretation of unloading slopes which assumes infinitesimal strains. The present paper deals with the way to be followed when classical measurements of elastic characteristics are not valid and have to be modified to take into account finite deformation. This is introduced through the consideration of a cylindrical sample subjected to a triaxial test which is assumed to preserve homogeneous state of stress and strain. In that case, the deformation gradient matrix can be easily expressed and incorporated in the calculation of Piola-Kirchhoff stress tensor in relation with Green-Lagrange strain tensor. Some results. obtained from experimental tests on typical high porosity isotropic chalks, are finally used to illustrate the necessary rectifications of Poisson ratio and elastic moduli measurements. 


\section{Introduction}

L'étude expérimentale de matériaux tendres tels que la craie poreuse peut parfois poser des problèmes de dépouillement dès que les déformations deviennent élevées. La légitimité de la détermination des caractéristiques élastiques en phase de déchargement par des considérations habituelles de linéarisation des déformations peut alors être mise en question. Il en est de même pour l'évaluation de la déformation plastique et de la porosité plastique [1] car la partition usuelle $(\bar{\varepsilon})=(\varepsilon)^{\rho}+(\tilde{\varepsilon})^{e}$ n'est plus valable dans le cadre des déformations finies. On peut dans ce cas passer par une configuration relâchée intermédiaire après déchargement [2] [3], qui n'est plus géométriquement confondue avec la configuration initiale. Pour un milieu poreux, cette configuration sera celle dans laquelle se trouve le matériau après un déchargement le ramenant aux conditions initiales de contraintes, température et pression interstitielle. Nous nous proposons ici d'étudier, dans le cadre d'essais triaxiaux (déviatoriques ou hydrostatiques), l'influence de cette déformation sur la mesure des coefficients élastiques et des déformations permanentes et commenter les écarts, par rapport à un dépouillement classique, avec quelques exemples obtenus sur une craie très poreuse. Les déformations pourront être alternativement mesurées par jauges d'extensométrie ou indirectement par capteurs de déplacement.

\section{2}

\section{Description de la déformation - Mesure de la déformation volumique pour la craie}

D'un point de vue expérimental, les déformations mesurées avec les dispositifs classiques d'extensométrie - jauges de déformation et capteurs LVDT - ont un caractère lagrangien si l'on prend comme référence la géométrie initiale de l'échantillon. Une jauge collée sur le matériau avant déformation donnera la déformation classique $\varepsilon=\frac{X-X}{X}, X$ longueur de référence de la configuration initiale et un capteur de déplacement permet d'obtenir $\mathrm{x}$-X que l'on pourra alors comparer à $X$ pour retrouver $\varepsilon$. De ces déformations, nous pourrons évaluer les changements de géométrie de l'échantillon et les différents gradients de transformation associés à ces changements. L'état de déformation de Green-Lagrange sera décrit localement par le tenseur $(\Delta)$. L'état de contraintes, sollicitant l'échantillon dans la configuration actuelle, est caractérisé par $(\overline{\bar{\sigma}})$, tenseur de contraintes de Cauchy. Un déchargement du matériau, restaurant l'ensemble des conditions initiales, donnera une réponse, supposée élastique, notée $(\bar{\varepsilon})^{\epsilon}$ et définie à partir de la configuration actuelle. Il est habituel de relier $(\bar{\sigma})$ à $(\bar{\varepsilon})^{e}$ pour évaluer les caractéristiques élastiques du matériau et ceci est légitime en petites perturbations. Si la géométrie de l'échantillon subit des transformations importantes, il faut revenir à une écriture objective de la loi de comportement en reliant $(\hat{\wedge})$ à $(\overline{\bar{\Pi}})$ tenseur de contraintes de Piola-Kirchhoff. Pour un matériau élastoplastique très déformable tel que la craie poreuse, il s'agira plus exactement de relier $(\overline{\bar{I}})$ à $(\wedge)^{\epsilon}$ partie élastique de la déformation de Green Lagrange relative à la configuration initiale. Les relations liant les teneurs $(\overline{\bar{\Pi}})$ à $(\hat{\bar{\Lambda}})^{\dagger}$ permettront alors la détermination des paramètres élastiques, indépendamment de l'état de déformation du matériau avant la décharge. On aura donc une mesure intrinsèque de ceux-ci. La figure 1, schématise les transformations géométriques locales, faisant apparaitre une configuration relâchée dont l'état de déformation est décrit par $(\bar{\Delta})$ f.

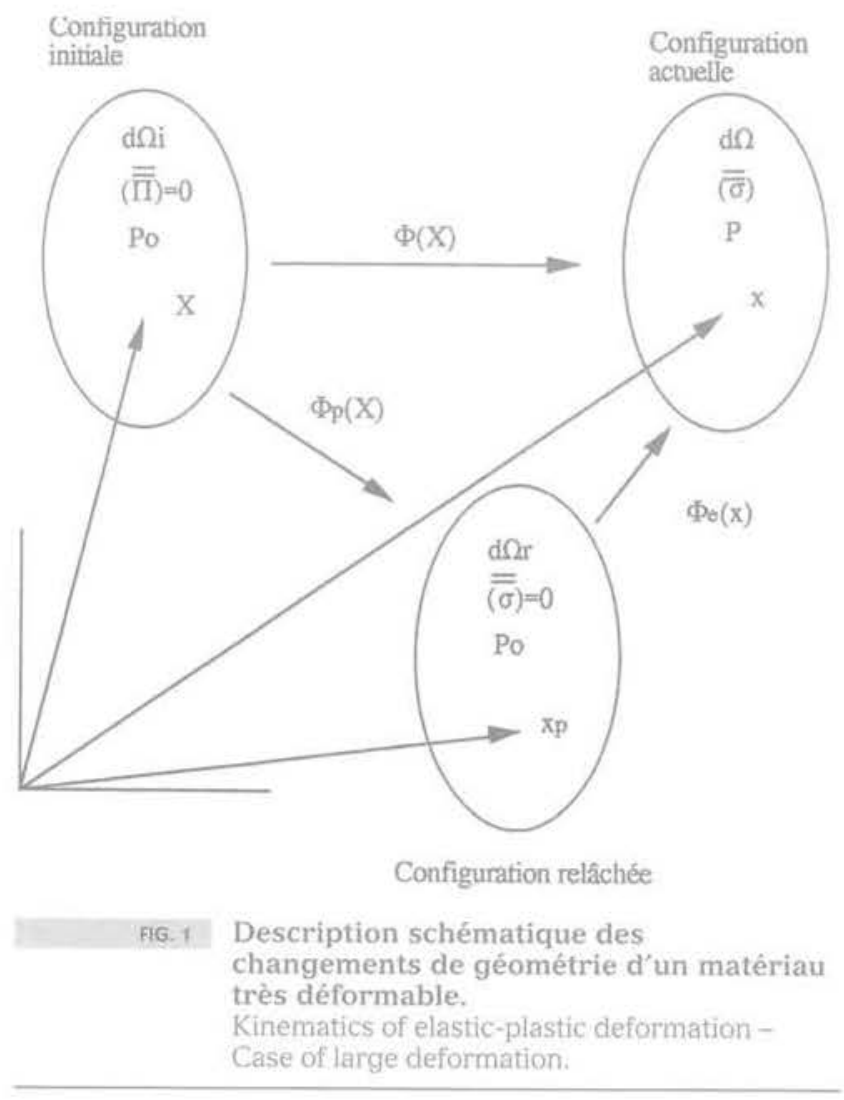

Conformément à cette représentation on peut écrire les transformations géométriques reliant les coordonnées matérielles $\mathrm{X}$ aux coordonnées spatiales $\mathrm{x}$ et $\mathrm{X}^{\mathrm{p}}$ par :

$$
\begin{aligned}
& X=\Phi(X) \\
& X^{p}=\Phi^{p}(X) \\
& X=\Phi^{e}\left(X^{p}\right) \\
& \text { ou } \\
& X=X^{p}+U^{e}\left(X^{p}\right) \\
& x^{p}=X-u^{e}(x)
\end{aligned}
$$

On peut supposer à ce stade que la transformation $\Phi^{e}$ obtenue après décharge élastique est une petite transformation et que les composantes du déplacement $\mathrm{U}^{e}$ ou $u^{e}$ ont la même écriture. Le tenseur de déformations élastiques écrit dans la configuration actuelle (et relâchée) sera donné par:

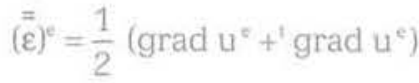

Le gradient est calculé par rapport aux variables $x$ ou $\mathrm{x}^{\mathrm{p}}$ et donne bien la déformation élastique relativement à la configuration actuelle (ou relâchée). Pour être 
traduit en variables de Lagrange ce tenseur doit être transporté dans la configuration initiale par la relation:

$$
\stackrel{=}{=}=\underset{(\Delta)^{\mathrm{e}}={ }^{\mathrm{t}}(\mathrm{F}) \cdot(\varepsilon)^{e} \cdot(\mathrm{F})}{=}
$$

$\stackrel{=}{(F)}$ est le gradient de la transformation $\Phi$ donné par:

$$
\mathrm{dx}=(\overrightarrow{\mathrm{F}}) \cdot \mathrm{dX}
$$

Si l'on note (P) gradient de la transformation plastique $\Phi^{p}$ tel que :

$$
\mathrm{dx}=(\stackrel{\overline{\mathrm{P}})}{\mathrm{p}} \cdot \mathrm{dX}
$$

alors le tenseur de déformations permanentes $(\Delta)^{\mathrm{P}}$ relatif à la configuration initiale est donné par:

$$
(\bar{\Delta})^{p}=\frac{1}{2}\left(^{+}(\overrightarrow{\mathrm{P}}) \cdot(\overrightarrow{\mathrm{P}})-(\overline{\mathrm{I}})\right.
$$

Le tenseur de déformations totales de GreenLagrange obtenu à partir de la transformation totale

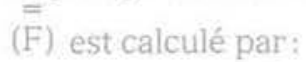

$$
(\bar{\Delta})=\frac{1}{2}\left({ }^{\prime}(\bar{F}) \cdot(\bar{F})-(\bar{I})\right)
$$

Il est donc bien clair ici que $(\bar{\Delta}) \neq(\bar{\Delta})^{e}+(\bar{\Delta})^{p}$. Ces transformations permettent également de rappeler la correspondance entre les tenseurs de Cauchy et PiolaKirchhoff:

$$
\operatorname{avec} \mathrm{J}=\operatorname{det}(\overrightarrow{\mathrm{F}})
$$$$
(\Pi)=J(F)^{-1} \cdot(\sigma)^{t}(F)^{-1}
$$

Si $\Omega, \Omega$ et $\Omega$ sont les volumes respectif́s des configurations initiales, actuelle et relâchée on aura:

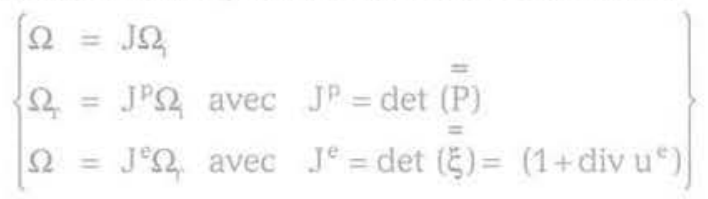

$(\zeta)$ est le gradient de la transformation $\Phi^{\mathrm{e}}\left(\mathrm{x}^{\mathrm{P}}\right)$.

Pour un matériau poreux saturé, la mesure de la porosité et de sa variation est essentielle. Comme l'a montré Coussy [1] la porosité n'est pas une variable d'état et c'est le contenu de la masse fluide fou de sa variation) qui est la variable appropriée de la description de l'évolution de l'espace poreux au cours du chargement. Dans le cas particulier d'une craie très poreuse, la matrice rocheuse (grains solides + porosité non connectée) peut être considérée comme incompressible élastiquement et plastiquement [4]. La mesure de la masse fluide, injectée ou expulsée par l'échantillon, au cours d'un essai, peut être valablement utilisée, comme nous le montrerons plus loin, pour estimer les variations de volume de l'échantillon. Cette mesure nous fournira alors la valeur des déterminants des gradients de déformation soient: J, Jp, Je. La figure 2 représente le cycle de chargement isotherme faisant varier l'état de contrainte total et le contenu en masse fluide.

$M_{\text {et }} M_{t}$ sont respectivement les masses volumiques de fluide par rapport au volume initial $\Omega$, injectées ou expulsées pendant la sollicitation. Les porosités seront notées $\phi_{0} \phi$ et $\phi_{\text {r }}$ pour les états initial, actuel et relâché. Dans le cas d'une déformation finie on ne peut pas calculer $\phi_{p}$ la porosité plastique par $\phi_{p}=\phi_{\mathrm{r}}-\phi_{0}$. En effet on $\mathrm{a}$ :

$$
M_{c}=J \rho^{n} \Phi-\rho_{0}^{n} \Phi_{\alpha}
$$

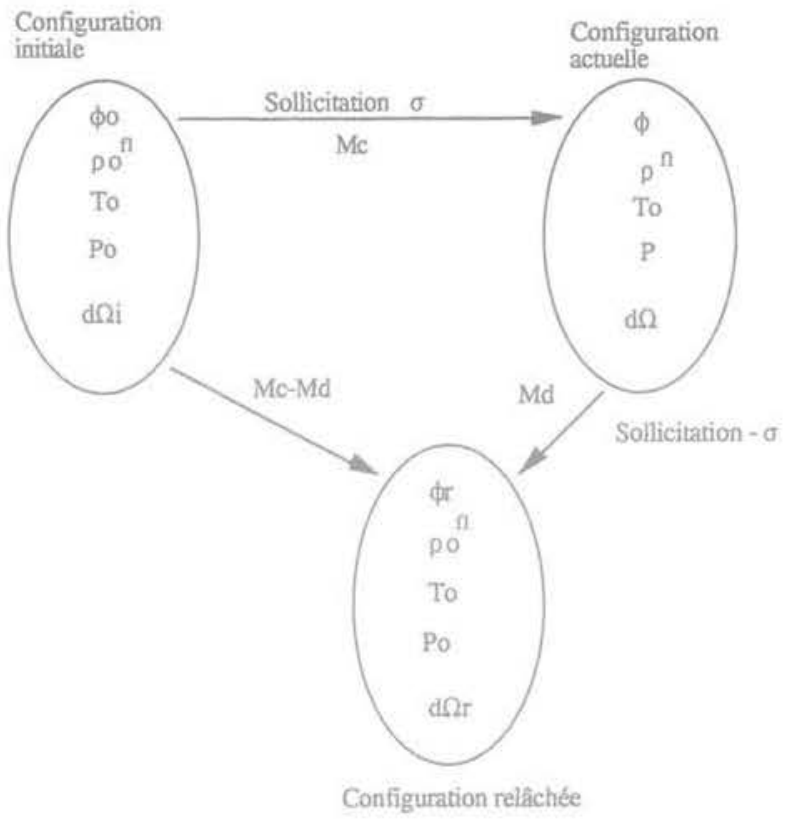

FIG. Q Variation irréversible de la porosité et du contenu de masse fluide au cours d'un cycle de chargement.

Irreversible mass fluid supply and porosity rate owing to a loading-unloading cycle.

$\rho^{f l}$ masse volumique du fluide à la pression $\mathrm{P}$.

$$
\left\{\begin{array}{l}
M_{c}-M_{d}=J p_{o}{ }^{n} \phi_{r}-p_{o}{ }^{f l} \phi_{o} \\
\text { et } \\
M_{c}-M_{d}=\rho_{o}{ }^{n} \phi_{p}
\end{array}\right.
$$

$M_{c}-M_{d}$ représente la variation irréversible de contenu en masse fluide. De ces relations on tire:

$$
\phi_{D}=J \phi_{1}-\phi_{0}
$$
nées

Cas particulier de la craie - conditions drai-

L'incompressibilité élastique et plastique de la matrice rocheuse, mentionnée auparavant, des craies très poreuses que nous avons testées permet l'obtention de quelques relations supplémentaires dont nous pourrons déduire une mesure des variations de volume de l'échantillon. Si l'on note $\varepsilon_{\mathrm{y}}=\frac{\mathrm{d} \Omega^{\mathrm{s}}-\mathrm{d} \Omega^{\mathrm{s}}}{\mathrm{d} \Omega^{\mathrm{s}}}$ la variation relative de volume de la matrice après la phase de chargement et $\varepsilon_{\mathrm{s}}^{p_{\mathrm{s}}}=\frac{\mathrm{d} \Omega^{\mathrm{s}} \mathrm{r}-\mathrm{d} \Omega^{\mathrm{s}}}{\mathrm{d} \Omega^{\mathrm{s}}}$ sa variation irréver sible de volume (dans la configuration relâchée), le calcul des porosités $\phi$ et $\phi_{\text {r }}$ donne:

$$
\left\{\begin{array}{l}
(J-1)(1-\phi)-\left(\phi-\phi_{0}\right)=\left(1-\phi_{0}\right) \varepsilon_{s} \\
\left(J^{\mathrm{P}}-1\right)\left(1-\phi_{\mathrm{r}}\right)-\left(\phi_{\mathrm{r}}-\phi_{\mathrm{o}}\right)=\left(1-\phi_{0}\right) \varepsilon^{\mathrm{p}} \mathrm{s}
\end{array}\right.
$$

Mais $\varepsilon^{p}=\varepsilon_{\mathrm{s}}=0$ et $\rho^{n}=\rho_{0}{ }^{n}$ car l'essai est drainé, on obtient donc en utilisant les équations (10), (11) et (13)

$$
\left\{\begin{array}{l}
\frac{M_{c}}{\rho_{0}^{n}}=J \phi-\phi_{0}=J-1 \\
\frac{M_{c}-M_{d}}{\rho_{0}^{\pi}}=J^{P} \phi_{r}-\phi_{0}=J^{P}-1
\end{array}\right.
$$


et également:

$$
J^{e}=\frac{J}{J^{p}}=\frac{M_{c}+p_{0}^{n}}{M_{c}-M_{d}+p_{o}^{n}}
$$

Les relations (14) et (15) montrent que nous pourrons déduire la déformation volumique du matériau par simple mesure de masse fluide expulsée ou injectée.

\section{3}

\section{Application à l'essai triaxial drainé}

L'essai triaxial conventionnel en cellule est fréquemment utilisé pour caractériser le comportement des milieux poreux. Il s'accompagne de mesures simultanées de masses fluides injectées ou extraites de l'échantillon [4] nécessaires à la mesure des variations de volume et de la porosité plastique $\phi$. Ce point est essentiel pour la modélisation poro-plastique de certaines roches. Nous allons cependant axer ici notre étude sur la mesure des paramètres élastiques d'un matériau très déformable, la craie, effectuée par un déchargement de l'échantillon après une déformation importante et dans le cas d'un essai drainé. Ce sont donc les paramètres élastiques dits « đrainés » qui seront introduits dans une méthode de correction des mesures prenant en compte la grande déformation du matériau. Nous supposerons que lors de cet essai (hydrostatique ou déviatorique), l'échantillon initialement cylindrique reste cylindrique. Les essais effectués sur ce matériau ont montré son comportement isotrope et, en petites perturbations, une relation élastique et linéaire entre le tenseur des contraintes de Cauchy (identique dans ce cas à celui de Kirchhoff) et le tenseur des déformations élastiques [4]. Nous supposerons alors, par extension, que la loi liant le tenseur des contraintes de Kirchhoff et le tenseur des déformations élastiques de Green-Lagrange est également linéaire. Dans la représentation de la figure 1, nous avons fait apparaître la configuration relâchée qui se déduit de la configuration actuelle par une déformation élastique. Cette configuration représente la position d'une particule d'un solide (l'échantillon) sollicité par un état homogène de contrainte et donc de déformation. L'opération de déchargement en contrainte de la surface de l'échantillon ramènera toutes les contraintes internes à une valeur nulle et il n'est donc pas nécessaire d'envisager, ici, de discontinuité dans la transformation géométrique décrivant le déplacement des particules de l'état relâché [5]. Ce point particulier serait à prendre en compte dans le cas d'une particule d'un solide soumis à un écoulement plastique non homogène et présentant un état de contraintes résiduelles après le déchargement. Par commodité nous utilisons un repérage cylindrique en notant $(R, \Theta, Z)$ les coordonnées initiales $(r, \theta, z)$ les coordonnées actuelles et $(\gamma, \varphi, \delta)$ les coordonnées de la configuration relâchée. La sollicitation est caractérisée par les contraintes totales principales de Cauchy: $\sigma_{2}=\sigma_{1}+\mathrm{P}$ et $\sigma_{f}=\sigma_{\mathrm{A}}=$ $\mathrm{P}_{\mathrm{e}}$. L'échantillon est pris comme volume élémentaire représentatif à l'échelle macroscopique du comportement du matériau, nous pouvons ainsi écrire la transformation géométrique $\Phi$ sous la forme:

$$
\left\{\begin{array}{l}
r=(1+\alpha) R \\
\theta=\Theta \\
z=(1+\beta) Z
\end{array}\right.
$$

avec $\alpha=\frac{\Delta\left(R_{0}\right)}{R_{0}}$ et $\beta=\frac{\Delta\left(H_{0}\right)}{H_{0}}$;

$\mathrm{R}_{\mathrm{o}}$ et $\mathrm{H}_{\mathrm{o}}$ sont les dimensions initiales, rayon et hauteur, de l'échantillon.

Le passage de la configuration actuelle à la configuration relâchée, représentant après plastification un retour élastique, sera décrit par:

$$
\left\{\begin{array}{l}
\gamma=\left(1+\alpha_{e}\right) r \\
\varphi=\theta \\
\delta=\left(1+\beta_{e}\right) z
\end{array} \quad \alpha_{e} \text { et } \beta_{e}\right. \text { petits }
$$

Notons que $\alpha_{e}$ et $\beta_{e}$ sont ici, $\frac{\Delta\left(r_{i n}\right)}{r_{n}}$ et $\frac{\Delta\left(h_{n}\right)}{h_{0}}$, $r_{i}$ et $h_{n}$ sont les dimensions actuelles (Fig. 3)

$\mathrm{Ro}$

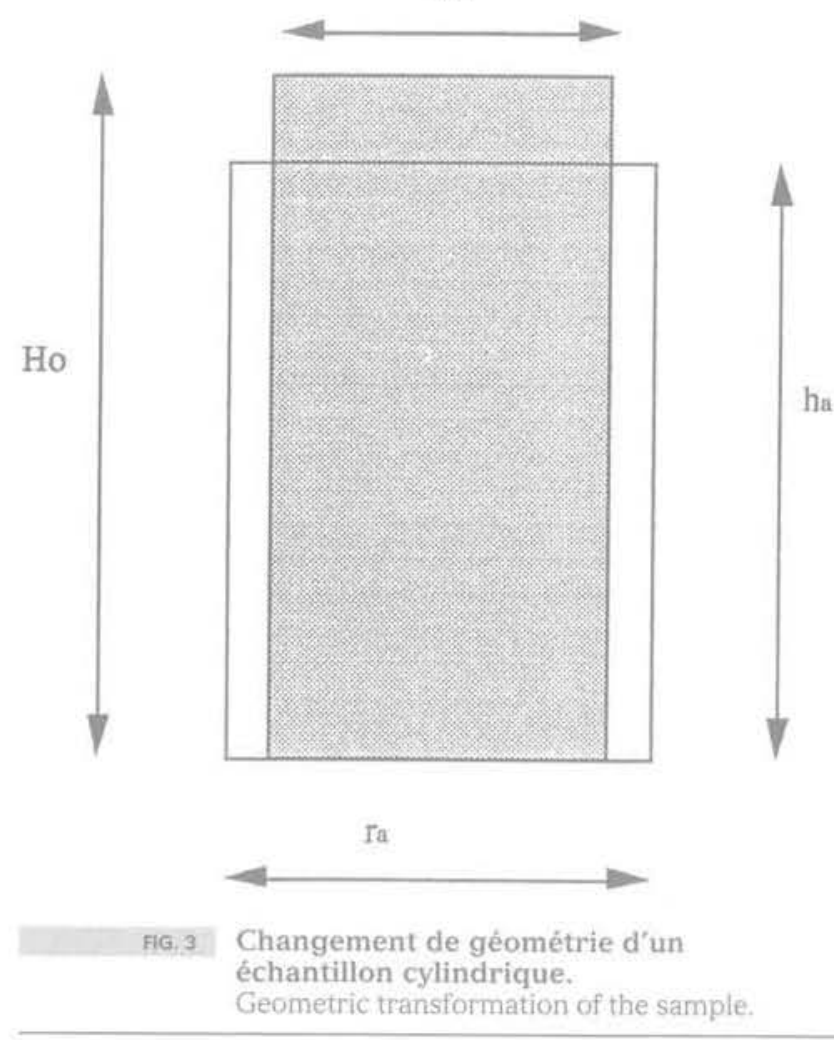

Le tenseur de déformations élastiques, exprimé dans la configuration relâchée s'écrira alors matriciellement par:

$$
=(\varepsilon)^{\mathrm{c}}:\left(\begin{array}{lll}
\alpha_{e} & 0 & 0 \\
0 & \alpha_{e} & 0 \\
0 & 0 & \beta_{e}
\end{array}\right)
$$

Sa transcription lagrangienne, effectuée par la

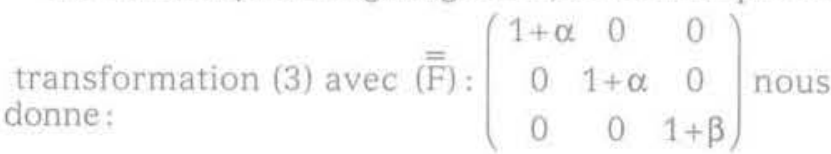

$$
(\bar{\Delta})^{e}:\left(\begin{array}{ccc}
(1+\alpha)^{2} \alpha_{e} & 0 & 0 \\
0 & (1+\alpha)^{2} \alpha_{e} & 0 \\
0 & 0 & (1+\beta)^{2} \beta_{e}
\end{array}\right)
$$


Ce tenseur est bien celui qu'il faut relier au tenseur de contraintes de Piola-Kirchhoff pour obtenir les caractéristiques élastiques du matériau. Ce tenseur, obtenu par la relation (8) s'écrit matriciellement:

$$
(\stackrel{=}{(\pi)}):\left(\begin{array}{ccc}
(1+\beta) P_{c} & 0 & 0 \\
0 & (1+\beta) P_{c} & 0 \\
0 & 0 & \frac{(1+\alpha)^{2}}{1+\beta} \sigma_{2}
\end{array}\right)
$$

Ces expressions montrent bien les corrections qu'il faut apporter aux différentes variables si on veut écrire rigoureusement les relations contraintes-déformations élastiques.

Avec les relations:

$$
\left\{\begin{array}{l}
\gamma=(1+\alpha)\left(1+\alpha_{e}\right) R \\
\varphi=\Theta \\
\delta=(1+\beta)\left(1+\beta_{e}\right) Z
\end{array}\right.
$$

on peut écrire le tenseur des déformations plastiques, en négligeant les termes élastiques du second ordre, sous la forme:

$$
\begin{gathered}
(\bar{\Delta})^{\alpha}:\left(\begin{array}{cc}
\alpha+\frac{\alpha^{2}}{2}+\alpha_{e}(1+2 \alpha) & \\
0 & \\
0 & 0 \\
\alpha+\frac{\alpha^{2}}{2}+\alpha_{e}(1+2 \alpha) & 0 \\
0 & \beta+\frac{\beta^{2}}{2}+\beta_{s}(1+2 \beta)
\end{array}\right)
\end{gathered}
$$

\section{4}

\section{Corrections sur les coefficients élastiques}

Nous allons plus particulièrement considérer les corrections sur la détermination des modules longitudinal et d'incompressibilité, $E_{b}$ et $K_{b}$ et du coefficient de Poisson $v_{b}$, relatifs au comportement drainé du matériau par rapport à une mesure "conventionnelle».

\section{1}

\section{Cas de l'essai hydrostatique}

Cet essai permet la détermination directe du module d'incompressibilité. Pour la craie de Lezennes testée dans la thèse de R. Kerbouche [4], la déformation volumique peut atteindre $12 \%$ à $\mathrm{P}_{c}=60 \mathrm{MPa}$. La figure 4 montre une large phase de compaction plastique dès que la pression de confinement excède un seuil de $20 \mathrm{MPa}$ environ.

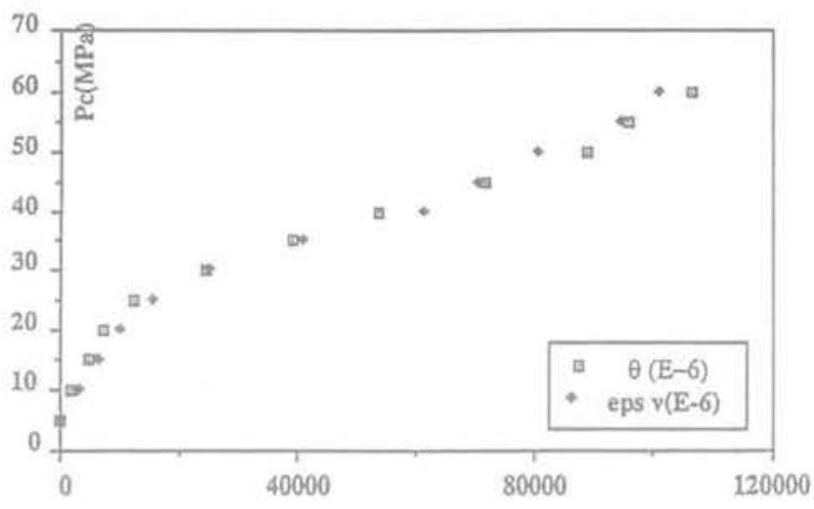

$\theta$ : variation volumique de masse fluide.

epsv: déformation volumique de l'échantillon (mesurée par capteur LVDT)

FiG:4

Essai hydrostatique drainé sur la craie de Lezennes.

Experimental results of drained hydrostatid test-Chalk from Lezennes.

Le souci de comparer les moyens de mesures lors du déchargement élastique nous conduit à considérer deux cas:

- la déformation est mesurée par jauges;

- la déformation est déduite d'une mesure de déplacement par capteurs LVDT et l'on mesure directement $\mathrm{u}(\mathrm{r})$ (ou $\Delta \mathrm{H})$.

Si la déformation est mesurée par jauge d'extensométrie, celle-ci indique la valeur après déchargement: $\varepsilon=\frac{u\left(r_{a}\right)}{R_{0}}$ alors qu'un capteur de déplacement donnera

$u\left(r_{0}\right)$. Dans une méthode de dépouillement classique, pour un matériau isotrope, on pose $\varepsilon_{\mathrm{v}}{ }^{\circ}=3 \varepsilon$ et $\mathrm{K}_{\mathrm{r}}$ module d'incompressibilité drainé, est déduit de $\mathrm{K}_{\mathrm{s}} \varepsilon_{\mathrm{c}}{ }^{\mathrm{c}}=\mathrm{P}$. En écriture lagrangienne les corrections vont intervenir à deux niveaux: sur la sollicitation $(\mathrm{P})$ et sur la déformation élastique. La relation (20) indique une correction par un facteur $(1+\alpha)$ sur la pression de confinement ( $\alpha=\beta$ car le matériau est isotrope). Nous écrivons alors pour déterminer $\mathrm{K}_{\mathrm{b}}$ :

$$
\mathrm{K}_{\mathrm{b}} \Delta_{\mathrm{v}}{ }^{\mathrm{c}}=\mathrm{P}_{\mathrm{c}}(1+\alpha)
$$

La relation (19) montre que $\Delta_{\mathrm{v}}{ }^{\mathrm{g}}=3(1+\alpha)^{2} \alpha_{\mathrm{e}}$ avec $\alpha_{\mathrm{e}}=$ $\alpha_{\mathrm{e}}=\frac{\mathrm{u}\left(\mathrm{r}_{\mathrm{a}}\right)}{\mathrm{r}_{\mathrm{H}}}$. Avec les relations (17) et (16) donnant $\mathrm{r}_{\mathrm{u}}=$ $(1+\alpha) R_{\alpha^{\prime}}$ l'égalité (23) devient:

$$
3 \mathrm{~K}_{\mathrm{b}}=\frac{\mathrm{u}\left(\mathrm{r}_{\mathrm{a}}\right)}{\mathrm{R}_{\mathrm{o}}}=\mathrm{P}_{\mathrm{c}}
$$

Ce résultat montre que, pour l'essai hydrostatique, l'exploitation «habituelle » des mesures ne nécessite pas de correction.

\section{2}

\section{Cas de l'essai déviatorique}

Le tableau I ci-dessous permet la comparaison directe des mesures que l'on effectue généralement (conventionnelles) et des mesures corrigées suite aux changements de géométrie de l'échantillon. Nous ne considérons ici qu'un retour élastique en ramenant le déviateur à une valeur nulle. La première correction affecte la contrainte $\sigma$, contrainte de Cauchy, dans le sens que l'on mesure le plus souvent l'effort de com- 


\begin{tabular}{|c|c|}
\hline Mesures conventionnelles & Mesures corrigée \\
\hline$\sigma_{1}($ conv $)=\frac{F_{1}}{S_{0}}$ & $\Pi_{z}=\frac{\sigma_{1}(\text { conv })}{1+\beta}$ \\
\hline$\varepsilon_{i}^{\epsilon}=\frac{\Delta\left(h_{n}\right)}{H_{n}}, \varepsilon_{q}^{\epsilon}=\frac{\Delta\left(r_{0}\right)}{R_{n}}$ & $\Delta_{t}^{e}=(1+\beta) \varepsilon_{1}^{e}, \Delta_{t}^{e}=(1+\alpha) \varepsilon_{t}^{e}$ \\
\hline $\mathrm{v}_{b}($ conv $)=-\frac{\varepsilon_{1}^{\mathrm{e}}}{\varepsilon_{i}^{\mathrm{e}}}$ & $v_{b}($ cor $)=\frac{1+\alpha}{1+\beta} v_{b}($ conv $)$ \\
\hline $\mathrm{E}_{\mathrm{b}}$ (conv) $=\frac{\sigma_{1} \text { (conv) }}{\varepsilon_{1}^{e}}$ & $E_{b}($ cor $)=\frac{\Pi_{2}}{\Delta_{1}^{e}}=\frac{\sigma_{1} \text { (conv) }}{(1+\beta)^{2} \varepsilon_{1}^{e}}$ \\
\hline
\end{tabular}

pression $\mathrm{F}_{1}$. La contrainte de Cauchy sera donc $\frac{\mathrm{F}_{1}}{\mathrm{~S}_{\mathrm{a}}}, \mathrm{S}_{4}$ étant la section actuelle de l'échantillon. Cette observation justifie la valeur de $\Pi_{2}$ donnée dans le tableau.

Afin d'évaluer l'importance des corrections nous pouvons considérer l'essai triaxial sur la craie de Liège [6] présentê par la figure 5 .

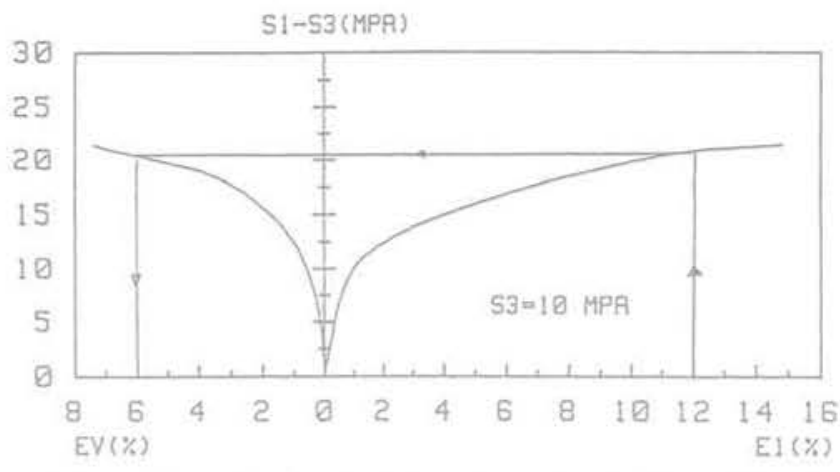

FG. 5 Essai triaxial sur la craie de Liège (d'après Shao [6]).

Experimental results of drained triaxial test Chalk from Liege.

La déformation volumique, mesurée par expulsion de fluide de l'échantillon, est de $6 \%$ environ et la déformation $\varepsilon_{2}=\varepsilon_{\text {c }}$ est de l'ordre de $12 \%$. Le volume de fluide expulsé, ramené au volume initial de l'échantillon, est égal dans ce cas à la variation lagrangienne de volume de l'échantillon soit $\frac{\Delta \Omega}{\Omega}$. On peut prendre pour estimer la correction à apporter aux résultats: $\mathrm{J}=0,94, \beta=$ $-0,12$ et $\alpha=0,033$ (par $\mathrm{J}=\operatorname{det}(\mathrm{F})$ ). Comme l'indique le tableau I, le facteur correctif du coefficient de Poisson est de $\frac{1+\alpha}{1+\beta}$. Ceci donne alors: $v_{b}($ cor. $) \approx 1,17 v_{b}$ (conv.). La correction sur le module longitudinal est plus élevée car le facteur correctif est ici de $\frac{1}{(1+\beta)^{2}}$ ce qui donne: $E_{j}$ (cor.) $\approx 1,29 E_{b}$ (conv.). Il faut enfin remarquer que les résultats présentés par la figure 5 sont obtenus sur un échantillon confiné initialement à $10 \mathrm{MPa}$. C'est cet état confiné qui est donc pris comme état de référence par rapport auquel les caractéristiques élastiques seraient déterminées. Si l'on voulait prendre comme référence le matériau à l'état vierge, il faudrait inclure, dans les coefficients $\alpha$ et $\beta$, les déformations obtenues pendant la phase de confinement. Cette prise en compte amplifierait en particulier la correction sur le module $\mathrm{E}_{\mathrm{b}}$.

\section{5}

\section{Conclusion}

Nous avons montré dans cette étude, illustrée par quelques exemples simples, que la détermination des caractéristiques élastiques d'un matériau, mesurées par une décharge élastique à l'issue d'une déformation importante (que l'on peut situer au-delà de $5 \%$ ), nécessite la prise en compte de cet état de déformation initiale dans l'exploitation des mesures. Les corrections à apporter sur celles-ci, si l'on veut être rigoureux dans l'écriture de la loi de comportement naturellement lagrangienne du matériau, peuvent parfois être considérables puisque de l'ordre de $30 \%$ dans certains cas de notre exemple. Pour calculer ces corrections nous avons utilisé la loi de comportement du matériau reliant le tenseur de déformation de Lagrange au tenseur des contraintes de Piola-Kirchhoff en faisant apparaître l'influence d'une déformation finie sur les composantes de ces tenseurs.

En conclusion, on ne peut qu'insister sur les précautions à prendre dans le dépouillement des essais si on désire déterminer avec rigueur les caractéristiques mécaniques de certains matériaux. Cet aspect peut s'avérer essentiel si l'essai concerne un matériau endommageable et dont le degré d'endommagement est décrit par la dégradation des propriétés élastiques de celui-ci. 
[1] Coussy O. - Mécanique des milieux poreux, Editions Technip, 1991.

[2] Lemaitre J., Chaboche J.-L. - Mécanique des matériaux solides, Dunod, 1985.

[3] Nguyen Q.S., Halphen B. - « Sur les lois de comportement élasto-visco-plastique à potentiel généralisé n. C. P. Acad. Sc Paris, t. 277. Sẻrie A. 1973, p. 319-322.

[4] Kerbouche R. - Etude expêrimentaje du comportement poro-élastoplastique de la craie, These de Doctorat, UST Lille, 1994.

[5] Lee E.H. - "Elastic-plastic deformation at finite strains n, Journal of Applied Mechanics, March 1969, p. 1-6.

[6] Shao J.-F, - a Etude du comportement d'une craie blanche très poreuse et modélisation 1 . Thèse de Doctorat, UST, Lille, 1987. 